\title{
The influence of advanced life support response time on patient outcomes after out-of-hospital cardiac arrest in Taipei.
}

Ying-Chih Ko ${ }^{1}$, Jen-Tang Sun ${ }^{23}$, Wen-Chu Chiang ${ }^{14}$, Shuo-Ting Hsu' ${ }^{2}$, Yu-Chun Chien ${ }^{5}$, Yao-Cheng Wang ${ }^{5}$, Ming-Ju Hsieh ${ }^{1}$, Jia-shan Chen ${ }^{2}$, Chih-Wei Yang ${ }^{16}$, Hui-Chih Wang ${ }^{1}$, Matthew Huei-Ming Ma ${ }^{14}$

1. Department of Emergency Medicine, National Taiwan University Hospital, Taipei City, Taiwan

2. Department of Emergency Medicine, Far Eastern Memorial Hospital, New Taipei City, Taiwan

3. Institute of Emergency and Critical Care Medicine, National Yang Ming University, Taipei City, Taiwan

4. Department of Emergency Medicine, National Taiwan University Hospital, Yun-Lin Branch C, Taiwan

5. The Emergency Medical Services(ambulance) Division, Taipei City Fire Department, Taiwan

6. Department of Medical Education, National Taiwan University Hospital, Taipei, Taiwan

\section{Background}

- Out-of-hospital cardiac arrests (OHCAs) remain a major event which affects public health worldwide.

- Emergency medical services (EMS) response time is one of prehospital factors associated with survival rate of patients with OHCA.

- The association between OHCA patient survival and ALS response time remained undetermined.

\section{Objective}

To determine whether shorter ALS response time was associated with improved chance of survival in non-trauma adult OHCA patients.

\section{Materials and methods}

- We analyzed Utstein-based registry data on OHCA patients in Taipei form 2011 to 2015.

- The eligible patients were adult, aged $\geqq 20$ years, with non-traumatic OHICA who underwent resuscitation attempts participated by ALS team.

- Exclusion criteria: Patients without complete data, witnessed by emergency medical technicians, or response time $\geqq 15$ minutes.

- The primary outcome was survival to discharge, and the secondary outcome is the favorable neurological outcome (cerebral performance category $\leqq 2$ ).

- Subgroup analyses were based on presenting rhythms of OHCA, including shockable, pulseless electrical activity (PEA) and asystole.

\begin{tabular}{|c|c|c|c|c|}
\hline & $\begin{array}{l}\text { Any ALS } \\
(n=4,278)\end{array}$ & ALS $(n=546)$ & $\begin{array}{c}\mathrm{ALS}+\mathrm{ALS} \\
(\mathrm{n}=115)\end{array}$ & $\begin{array}{l}A L S+B L S \\
(n=3,617)\end{array}$ \\
\hline \multicolumn{5}{|c|}{ Patient } \\
\hline Age, years(median[Q1-Q3]) & $78(64,86)$ & $77(65,85)$ & $71(61,82)$ & $78(64,86)$ \\
\hline Male, number(percent) & $2652(61.99 \%)$ & 342 (62.64\%) & $80(69.57 \%)$ & $2230(61.65 \%)$ \\
\hline Witness, number(percent) & $1248(29.17 \%)$ & 188 (34.43\%) & $50(43.48 \%)$ & $1010(27.92 \%)$ \\
\hline Bystander CPR, number(percent) & 1669 (39.01\%) & 192 (35.16\%) & $28(24.35 \%)$ & 1449 (40.06\%) \\
\hline Shockable rhythm, number(percent) & $436(10.19 \%)$ & $68(12.45 \%)$ & $20(17.39 \%)$ & 348 (9.62\%) \\
\hline \multicolumn{5}{|c|}{ Location of arrest, EMS time interval and treatment (median[Q1-Q3] or \%) } \\
\hline Public, number(percent) & $326(7.62 \%)$ & 74 (13.55\%) & $18(15.65 \%)$ & $234(6.47 \%)$ \\
\hline Response time, minutes(median[Q1-Q3]) & $9(7,12)$ & $6(4,7)$ & $5(4,7)$ & $10(8,12)$ \\
\hline Scene time, minutes(median[Q1-Q3]) & $15(13,18)$ & $15(13,18)$ & $17(15,21)$ & $15(12,17)$ \\
\hline Medical center, number(percent) & 2125 (49.67\%) & $294(53.85 \%)$ & 66 (57.39\%) & $1765(48.8 \%)$ \\
\hline Adrenaline, number(percent) & 1366 (31.93\%) & $214(39.19 \%)$ & 66 (57.39\%) & $1086(30.02 \%)$ \\
\hline $\begin{array}{c}\text { Atropine/amiodarane/others, } \\
\text { number(percent) }\end{array}$ & $57(1.33 \%)$ & $14(2.56 \%)$ & $3(2.61 \%)$ & $40(1.11 \%)$ \\
\hline Endotracheal tube, number(percent) & 789 (18.44\%) & 161 (29.49\%) & 34 (29.57\%) & 594 (16.42\%) \\
\hline \multicolumn{5}{|c|}{ Survival status, number(percent) } \\
\hline Any ROSC & 1149 (26.86\%) & $187(34.25 \%)$ & 40 (34.78\%) & $922(25.49 \%)$ \\
\hline Sustained ROSC & 993 (23.21\%) & 162 (29.67\%) & $37(32.17 \%)$ & 794 (21.95\%) \\
\hline Survival to discharge & $287(6.71 \%)$ & $51(9.34 \%)$ & $18(15.65 \%)$ & $218(6.03 \%)$ \\
\hline $\mathrm{CPC} \leqq 2$ at discharge & $126(2.95 \%)$ & $26(4.76 \%)$ & $10(8.7 \%)$ & 90 (2.49\%) \\
\hline
\end{tabular}

\section{Result}

- A total of 4,278 cases were included in final analysis. The median ALS response time was 9 minutes (interquartile range 7 to 12 ).

- Every minute delay of any ALS response time would reduce $7 \%$ chance of survival to discharge (adjusted odds ratio [aOR] 0.93; 95\% confidence interval [Cl]: 0.90-0.97) as well as favorable neurological outcome (aOR 0.91; 95\%Cl: 0.86-0.97).

- Subgroup analysis showed the longer ALS response time was negatively associated with the chances of survival to discharge among OHCA patients with shockable rhythm (aOR 0.89; 95\% $\mathrm{Cl}: 0.83-0.95)$ and PEA group (aOR 0.92; 95\%Cl: 0.86-0.99).

\section{Conclusion}

In non-trauma adult OHCA in Taipei, the longer ALS response time was associated with the worse odds of survival to discharge and favorable neurologic outcome, especially in patients with presenting shockable rhythm and PEA group.

\begin{tabular}{|c|c|c|c|c|}
\hline & $\begin{array}{c}\text { Any ALS } \\
\text { OR(CI), n }\end{array}$ & $\begin{array}{c}\text { ALS } \\
\mathrm{OR}(\mathrm{Cl}), \mathrm{n} \\
\end{array}$ & $\begin{array}{l}\text { ALS+ALS } \\
\text { OR(CI), n }\end{array}$ & $\begin{array}{l}A L S+B L S \\
O R(C), n \\
\end{array}$ \\
\hline \multicolumn{5}{|l|}{ Survival to discharge } \\
\hline Shockable +non-shockable & $\begin{array}{ll}0.93 & 4,278 \\
(0.90,0.97)\end{array}$ & $\begin{array}{l}0.94 \quad 546 \\
(0.81,1.09)\end{array}$ & $\begin{array}{ll}0.98 \quad 115 \\
(0.71,1.35)\end{array}$ & $\begin{array}{ll}0.94 & 3,617 \\
(0.90,0.98)\end{array}$ \\
\hline $\mathrm{pVT} / \mathrm{Vf}$ & $\begin{array}{lc}0.89 & 436 \\
(0.83, & 0.95)\end{array}$ & $\begin{array}{cc}0.77 \quad 68 \\
(0.55,1.08)\end{array}$ & $\begin{array}{ll}\text { NA } & 20 \\
\end{array}$ & $\begin{array}{ll}0.89 & 348 \\
(0.83, & 0.96)\end{array}$ \\
\hline Non-shockable rhythm & $\begin{array}{cc}0.95 & 3,842 \\
(0.91,1.002)\end{array}$ & $\begin{array}{ll}0.96 & 478 \\
(0.80,1.15)\end{array}$ & $\begin{array}{l}1.02 \quad 95 \\
(0.71,1.49)\end{array}$ & $\begin{array}{lr}0.97 & 3,269 \\
(0.92,1.02)\end{array}$ \\
\hline PEA & $\begin{array}{lr}0.92 & 800 \\
(0.86, & 0.99) \\
\end{array}$ & $\begin{array}{ll}0.81 & 126 \\
(0.59,1.11)\end{array}$ & NA 28 & $\begin{array}{ll}0.93 & 646 \\
(0.85,1.01)\end{array}$ \\
\hline Asystole & $\begin{array}{ll}0.99 & 3,018 \\
(0.93,1.06)\end{array}$ & $\begin{array}{lr}1.09 & 348 \\
(0.87,1.37) \\
\end{array}$ & NA & $\begin{array}{lr}1.01 & 2,603 \\
& (0.95,1.08) \\
\end{array}$ \\
\hline \multicolumn{5}{|l|}{ CPC 1 or 2} \\
\hline All & $\begin{array}{ll}0.91 & 4,278 \\
(0.86,0.97)\end{array}$ & $\begin{array}{lr}0.81 & 546 \\
(0.62,1.05)\end{array}$ & $\begin{array}{ll}1.19 & 115 \\
(0.72,1.97)\end{array}$ & $\begin{array}{ll}0.93 & 3,617 \\
(0.87,0.99)\end{array}$ \\
\hline $\mathrm{pVT} / \mathrm{Vf}$ & $\begin{array}{lr}0.88 & 436 \\
(0.81,0.95) \\
\end{array}$ & $\begin{array}{l}0.82 \quad 68 \\
(0.6,1.13) \\
\end{array}$ & NA $\quad 20$ & $\begin{array}{lr}0.90 & 348 \\
(0.81, & 0.99) \\
\end{array}$ \\
\hline Non-shockable rhythm & $\begin{array}{ll}0.95 & 3,842 \\
(0.87,1.04)\end{array}$ & $\begin{array}{l}0.71 \quad 478 \\
(0.41,1.21)\end{array}$ & $\begin{array}{l}2.0195 \\
(0.56,7.27)\end{array}$ & $\begin{array}{lr}0.96 & 3,269 \\
(0.87,1.07)\end{array}$ \\
\hline PEA & $\begin{array}{ll}0.90 & 800 \\
(0.80,1.01)\end{array}$ & $\begin{array}{ll}0.69126 \\
(0.38,1.26)\end{array}$ & NA 28 & $\begin{array}{lr}0.89 & 646 \\
(0.78,1.03)\end{array}$ \\
\hline Asystole & $\begin{array}{ll}1.07 & 3,018 \\
(0.94,1.20)\end{array}$ & $\begin{array}{ll}\mathrm{NA}^{* *} \quad 348 \\
\end{array}$ & $\mathrm{NA}^{* *}$ & $\begin{array}{ll}1.05 & 2,603 \\
(0.92, & 1.19)\end{array}$ \\
\hline
\end{tabular}

Table 2. Logistic regression analysis 